\title{
BMJ Open The involvement of early stage breast cancer patients during oncology consultations in Italy: a multi-centred, randomized controlled trial of a question prompt sheet versus question listing
}

\author{
Alessandro Bottacini, ${ }^{1}$ Claudia Goss, ${ }^{1}$ Maria Angela Mazzi, ${ }^{1}$ Alberto Ghilardi, ${ }^{2}$ \\ Chiara Buizza, ${ }^{2}$ Annamaria Molino, ${ }^{3}$ Elena Fiorio, ${ }^{3}$ Rolando Nortilli, ${ }^{4}$ Vito Amoroso, ${ }^{5}$ \\ Lucia Vassalli, ${ }^{5}$ Richard F Brown ${ }^{6}$
}

To cite: Bottacini A, Goss C, Mazzi MA, et al. The involvement of early stage breast cancer patients during oncology consultations in Italy: a multi-centred, randomized controlled trial of a question prompt sheet versus question listing. BMJ Open 2017;7:e015079. doi:10.1136/ bmjopen-2016-015079

- Prepublication history and additional material are available. To view these files please visit the journal online (http://dx.doi org/10.1136/bmjopen-2016015079).

Received 7 November 2016 Revised 8 April 2017 Accepted 21 April 2017

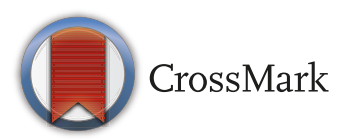

For numbered affiliations see end of article.

Correspondence to Dr Alessandro Bottacini; alessandro.bottacini@univr.it

\section{ABSTRACT}

Objectives To investigate, prior to an oncology consultation, the use of a pre-prepared list of evidence based questions, Question Prompt Sheet (QPS), compared with a Question List (QL), a patient self-generated list of questions.

Design Multi-centred, randomised controlled trial. Setting Secondary-care patients attending three outpatient oncology clinics in Northern Italy.

Participants 308 women completed the study. Inclusion criteria were an age between 18 and 75 years, a recent diagnosis of early stage, non-metastatic breast cancer, adequate Italian language skills, no previous oncology visits and no evidence of cognitive impairment.

Intervention Patients received the QPS or the QL prior to the consultation, completed it without suggestion or coaching session and delivered back before the visit.The consultations were audio-recorded and analysed for the number and content of questions. Multilevel linear models were used to compare the two groups.

Outcome measures The primary outcome was the comparison of questions asked between QPS and QL group Secondary outcomes included satisfaction about questions asked, satisfaction with decision, and level of anxiety. Results Patients in the QPS and QL group asked 13 and 16 questions respectively. The difference was not significant $(b=1.7, \mathrm{Cl}-0.3$ to $3.6, p=0.10)$. A mean of 22 questions was selected in the QPS, while a mean of 2 questions was written in the QL. Patients in the QPS group were significantly less satisfied $(t=3.60, p<0.01)$ with questions asked but wanted less additional information $(t=2.20, p<0.05)$. Levels of patient decisional satisfaction were equivalent between groups. Similarly, anxiety levels were equal between groups prior to the consultation and decreased in similar way after the consultation.

Conclusions Both interventions have similar impact on patients' participation in terms of question asking during the consultation. Future research is needed in order to explore which components of the interventions are really useful and efficacious.

\section{Strengths and limitations of this study}

- This is the first trial in Europe comparing two different methods of promoting question asking prior to an oncology consultation.

- The strength of the study lies in the use of a rigorous randomised controlled methodology and, differently from previous studies, oncologists were blinded to which experimental group to which participants were randomised thus allowing the evaluation of the real power of the intervention.

- In order to ensure that the oncologists were blind to their patient experimental group assignment, copies of the completed QPS or QL were not provided for patients to use during their consultation to serve as a reminder, so it may be that the number of questions asked could be mediated by the patient's recall ability.

Trial registration ClinicalTrials.gov NCT01510964

\section{INTRODUCTION}

In recent years, the patient-physician relationship has been evolving largely due to changes in patient preferences for more information ${ }^{1}$ and a more active role in the decision making process regarding their illness.

Patient centred communication has become the gold standard for excellence in clinical communication. It consists of clinicians eliciting and understanding their patients' perspectives and social contexts, reaching a shared understanding and empowering patients by offering meaningful involvement in treatments decisions. ${ }^{2}$ 
One approach to promote patient-centred communication is to provide mechanisms to involve and support active participation in consultations, by encouraging patients to ask questions. Asking questions can be considered a first index of active participation in the discussion and an expression, at the same time, of a patient's most immediate information needs. ${ }^{3-7}$ Asking questions can lead to positive benefits for cancer patients, including greater satisfaction and better treatment adherence. ${ }^{89}$

Two main techniques have been tested in the oncology setting to promote question asking. First, Question Listing (QL), before the visit with their oncologist, patients have been encouraged to generate their own list of questions ${ }^{10}$ about their cancer and treatment options through a coaching process with an independent assistant. ${ }^{1-13}$ A consultation plan is then generated and given to the patient and the oncologist to be used as a visual aid during the consultation. ${ }^{14}$ Second a Question Prompt Sheet QPS), before a consultation with their oncologist, patients have been provided with a pre-prepared list of evidence based questions, a QPS that prompt patients to consider novel topics before they see their oncologist and serves to remind patients to raise these questions with their oncologist during their consultation. ${ }^{15-19}$ In the oncology setting, using the QPS coupled with oncologist endorsement of question asking, had shown that: (a) patients were encouraged to ask more questions, in particular about sensitive and difficult topics such as prognosis, diagnosis, and issues surrounding end of life care ${ }^{151718}$; (b) the quality of the information exchanged between the physicians and patients had been increased, ${ }^{45-17}$ and (c) patient outcomes had been improved (eg, enhanced information recall and reduced level of anxiety during the consultation). ${ }^{1517182021}$

In this Randomised Controlled Trial (RCT) we assessed whether there was a difference in the efficacy of a Question Prompt Sheet (QPS) versus a Question Listing (QL) method to increase the number of questions that breast cancer patients with early stage disease asked during their initial encounter with an oncologist. We also assessed the differential impact of each method on patient outcomes. This study is innovative in three ways. First, this study compared the efficacy of the two interventions with each other while previous studies have compared either the QPS or QL, with a standard of care control group, which risks overestimating the real effect of the intervention. ${ }^{10-13}$ 15-19 Second, moreover, the oncologists in this study were blind to their patients' treatment group allocation. In order to achieve this, both the completed QPS and QL were collected just before the patient visit. This approach enabled the evaluation of the real power of the interventions and guarded against the potential for Hawthorne effects. ${ }^{22}$ Third, patients in the QL group in this study generated their questions alone without coaching or other assistance.

The QPS is a more structured intervention in that patients receive many previously prepared, evidence based questions in several topic areas that can be selected and asked during the consultation. On the other hand, the QL intervention requires greater patient effort as patients must generate their own question and, in the process, they may focus on a limited range of their most immediate information needs. For this reason we hypothesised that the use of the QPS, prior to an oncology consultation, could further increase the number of questions asked in general and by specific topic areas (eg, prognosis) compared with the use of the QL. We also hypothesised that the use of the QPS would result in; a) greater patient satisfaction about the information, b) greater patient satisfaction with the treatment decision and c) less patient anxiety after the consultation compared with the use of the QL.

\section{METHODS}

\section{Study design}

This study was a multi-centred randomised controlled trial in which patients were randomised to the QPS or $\mathrm{QL}$ in a 1:1 ratio. The randomization sequences were generated off-site and stratified by centre by the study statistician (MAM). These sequences remained unknown to the research assistants involved in the data collection phase. Both the randomization procedure and the treatment allocation techniques were developed to fully conceal the allocation from the research assistants, patients and oncologists. First, patients were given the intervention, either the QPS or QL, in a sealed envelope. Once the QPS or QL were completed they were placed into the same envelope, resealed and delivered back to the research assistant (RA). Second, patients were unaware of the detailed contents of the treatment that they did not receive, thus patients allocated to the QPS did not know the content of the QL intervention. Third, Oncologists did not know what type of intervention had been completed by the patients before the visit.

The study followed CONSORT guidelines ${ }^{23}$ and was approved by the Ethics Committee of the Hospital Trust of Verona and by the Provincial Ethics Committee of Brescia. The study protocol has been published elsewhere. $^{24}$

\section{Patients and recruitment}

The patient participants were all women with early stage breast cancer. Physician participants were the oncologists treating these patients in one of three outpatient clinics in Northern Italy run by the Hospital Trusts of Verona and Brescia. ${ }^{24}$

To be eligible patients were aged between 18 and 75 years, had a recent diagnosis of early stage, non-metastatic breast cancer (from stage 0 (Tis) to stage III), had adequate native language skills to complete questionnaires, had not visited any oncologist previously and no evidence of cognitive impairment (patients who had severe psychiatric or neurological diagnosis that could have an impact to cognitive functions were excluded). 
At study commencement oncologists provided written informed consent and provided demographic data that included their gender, age and years of oncology experience. Eligible patients were approached in the clinic waiting room by the oncology nurse who described the study goals and elicited the patient's interest in participating. Interested patients were accompanied to a dedicated room and were given detailed information by the research assistant. Willing patients provided written informed consent to participate in the study including consultation audio recording. Patients were then randomised to one of the two intervention groups within each centre.

Group 1 - Question Prompt Sheet (QPS) - Patients were provided with a Question Prompt Sheet.

Group 2 - Self generated Question List (QL) - Patients were provided with a blank lined form to write their own questions.

Patients completed the QPS or the QL in the clinic room in privacy prior to their appointment with their oncologist. They were not given a time limit to complete either intervention.

Recruitment was conducted between June 2011 and May 2013.

Socio-demographic data, such as age, marital status, education and employment were collected before the randomization along with pre-consultation levels of anxiety. All consultations were audio-recorded to allow an analysis of the consultation length and of the number of questions asked by the patient. Immediately following the consultation patients completed three questionnaires that measured post consultation levels of anxiety, satisfaction with information and satisfaction with the decision.

\section{Question counting}

Patients' questions were defined as 'utterances in interrogative form that ask for information or clarification'. ${ }^{25}$ Six categories were identified, five derived from those described in the literature ${ }^{115}$ (symptoms, aetiology, prognosis, prevention and illness management), and one specifically added (administrative questions). A codebook was developed that provided definitions, examples and decision rules to guide question classification, by each category. Inter-rater reliability was calculated among the three coders was calculated based on ten consultations. Three coders were utilised due to volume of transcripts generated by the study requirements. The results revealed an average percent agreement of $62.9 \%$ and an acceptable Cohen's kappa of 0.49.

Subsequently, all interviews were equally distributed among the three coders who listened to the audiotapes, identified all direct patients' questions, transcribed them verbatim and categorised them using the categories previously identified.

The primary outcome was the total number of questions asked and the number of questions within each of these content categories. ${ }^{23}$

\section{Materials}

\section{Question Prompt Sheet (QPS)}

The QPS is a structured list of 50 evidence-based questions that patients commonly want to ask their oncologist. The original English QPS developed by the Australian group $^{3}{ }^{15-18} 26$ and largely used both for research and clinical purposes was translated and adapted for Italian patients in collaboration with the senior author (RB) ${ }^{27}$

Participants were asked to circle salient questions, if any, they would like to ask their oncologist. The main topic areas regarded the diagnosis, the prognosis, the treatment options and some other additional information (eg, costs, support information).

\section{Self-generated Question List (QL)}

The QL form is a blank lined paper where participants were asked to independently self-generate a list of questions they would like to ask their oncologist without any previously coaching session. Participants were instructed to:

'Please indicate the issues which you want to discuss today with your oncologist and the questions you want to ask'.

\section{Questionnaires}

Satisfaction with information was measured by a study specific questionnaire composed by the three following questions:

\section{a) "How much have you been able to ask those questions you} had previously selected or written?, b) 'How much did the oncologist answer in a clear and comprehensive way?' and

c) 'How much do you feel the need for further information?".

Responses were recorded on Likert scales with anchors at

$$
\text { ' } 1 \text { - not at all' and '5 - very much so'. }
$$

Satisfaction with Decision was measured using the Satisfaction With Decision scale (SWD) ${ }^{28}$ a six item questionnaire with patients responses reported on a Likert scale with anchors at

$$
\text { '1 - strongly disagree' and '5 - strongly agree'. }
$$

The state anxiety level before the consultation was assessed using the Spielberger State-Trait Anxiety Inventory-X1 (STAI-X1), ${ }^{29}$ a validated, widely used measure that consists of 20 items with responses reported on a Likert scale with anchors at

\section{'1 - not at all' and ' 4 - very much so'.}

The state anxiety level after the consultation was assessed using the STAI-X1/R, ${ }^{30}{ }^{31}$ which is a ten-item reduced version of the STAI-X1, with responses reported in an identical manner.

Anxiety fluctuation before and after the consultation was calculated by the difference between the two total scores on the 10 items common in both scales. The value obtained is called STAI-DIFF. ${ }^{30} 31$ 
See Appendix A for a copy of questionnaires used.

\section{Sample size calculation and statistical analysis}

The sample size calculation utilised indicated that we need a sample of 300 participants, so that the 50 patients in each arm, within the three centres. This number was estimated assuming a clinically relevant difference of 5 questions between QPS and QL group (30\% effect size). No significant differences in question asking were expected between the three centres. The unpaired t-test had a power of $80 \%$, a significance level of $5 \%$ while taking into account a withdrawal rate of $15 \%$ (more details in the protocol) ${ }^{24}$

The difference in the number of patient questions within the two intervention groups (Aim 1) was assessed by a multilevel linear regression. The regression considered the hierarchical design (patients nested into doctors and doctors into centres) ${ }^{32}$ and was adjusted for confounders, introduced as covariates in the model. A preliminary exploration using a Pearson's correlation coefficient found that consultation length and presence of a companion were significantly correlated with the dependent variable (number of questions). Due to a non normal distributions, a Wilcoxon non-parametric test was conducted to to explore differences between question topics desired by patients between experimental groups.

For Aim 2, after having checked for the presence of socio-demographic differences among centres, as potential confounders (with chi-squared test and one-way ANOVA, performed for categorical and continuous variables respectively), Student's $t$-tests were carried out to explore between group differences in satisfaction with information, satisfaction with decision and pre/post consultation levels of anxiety.

A data set containing the study data is available in PDF format as supplementary material .

\section{RESULTS}

Twenty oncologists were recruited, who were mostly female $(85 \%)$ with an average age of 38.8 years and an average 11.3 years of experience as a medical oncologist. Each oncologist conducted an average of 15 consultations for the study ( $\mathrm{SD}=16.9$, range 1-52).

Three hundred and twenty-four patients participated in the study. One hundred and sixty-four were randomised to the QPS group and the remainder to the QL group (figure 1). Sixteen audio-recordings were drop out due to technical issues. Three hundred and eight female patients with a mean age of 55.6 years were finally analysed. The average time since diagnosis was 2 months.

Patient demographic variables are shown in table 1 . Differences between groups on these variables were non-significant, indicating that the two groups are similar with respect to the above characteristics.

\section{Consultation characteristics}

The length of the consultations ranged between 12 and 129 min with an average of $49 \mathrm{~min}(\mathrm{SD}=19)$. A family member was present in $72 \%$ of consultations for both the the QPS and QL groups.

The correlation between the consultation length and number of questions was $0.45(\mathrm{p}<0.01)$ while the correlation between the presence of family member and the

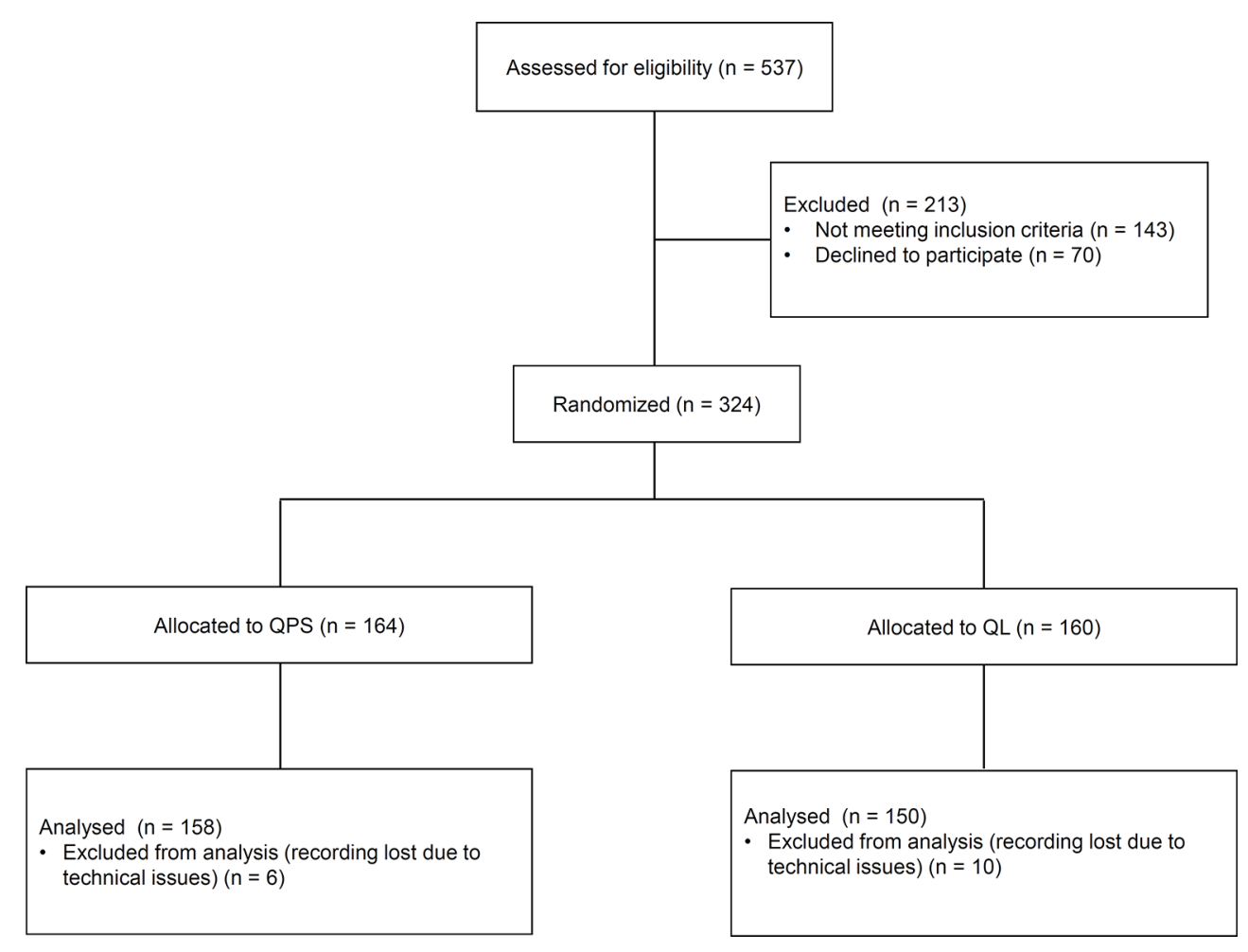

Figure 1 CONSORT diagram. QL, question list; QPS, question prompt sheet. 


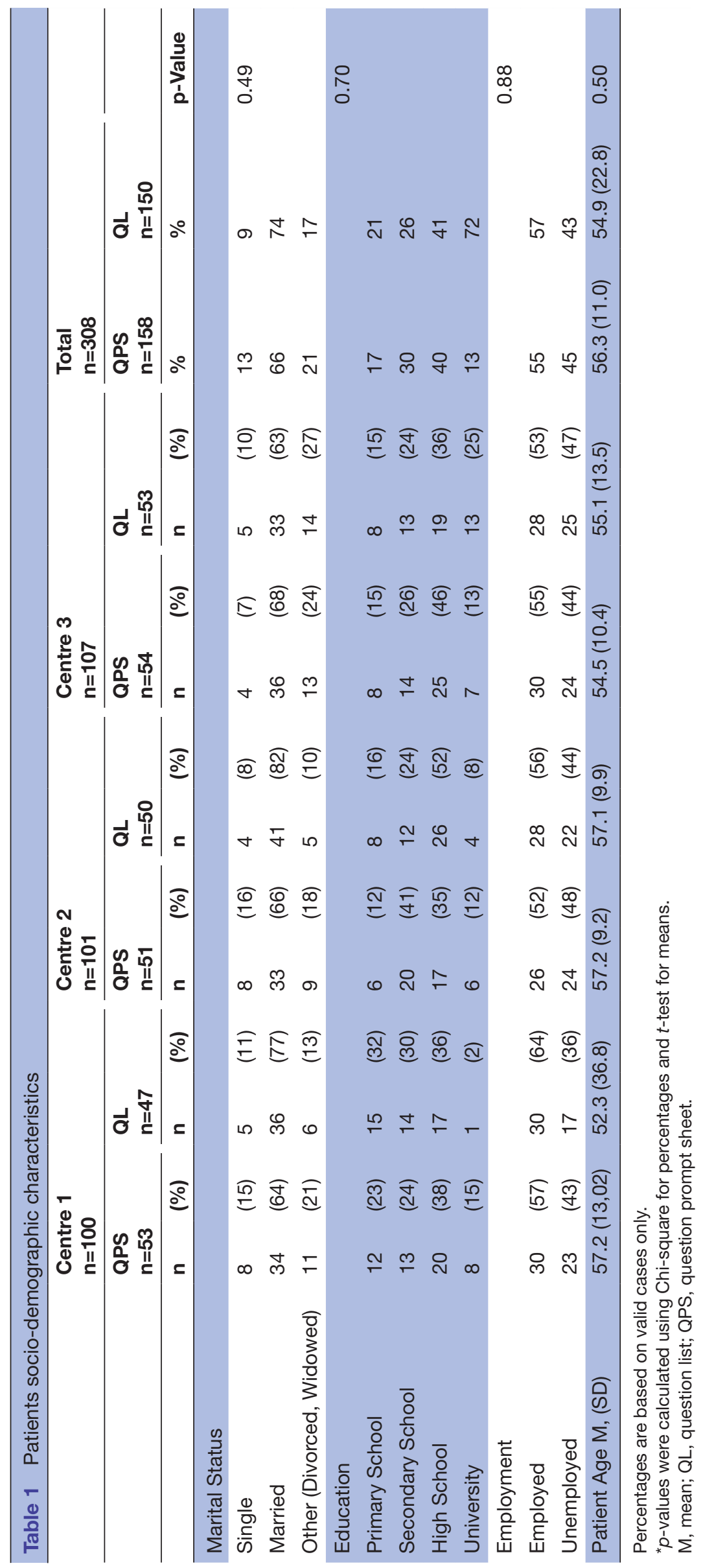

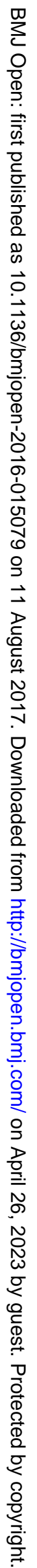



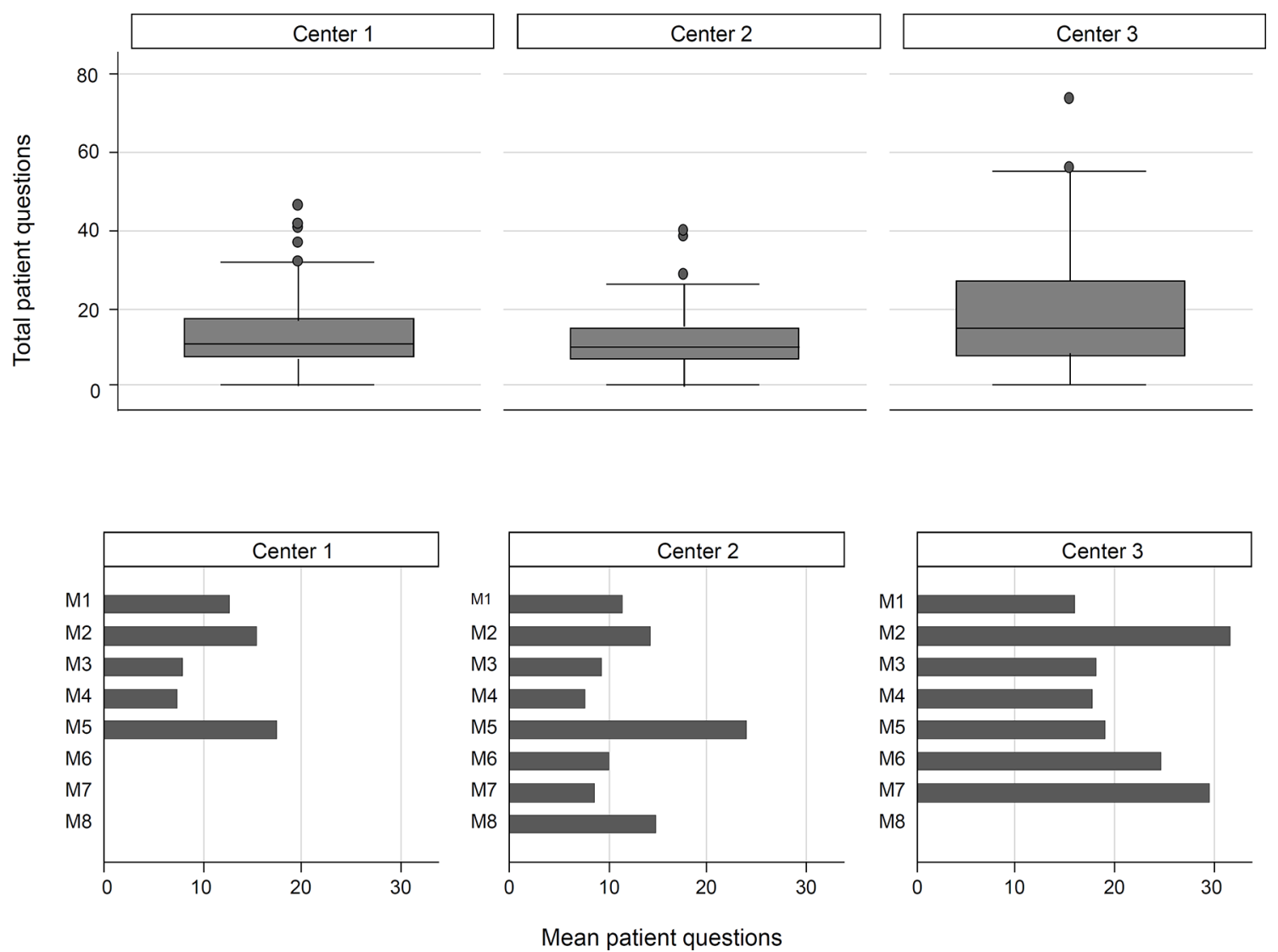

Figure 2 Number of patient's questions per interview. The box-plot graphs show the frequency distribution within centres and bar graphs show the mean values of patients' questions per doctor, nested in centres.M1, M2 etc.: medical oncologist.

number of questions was $0.24(\mathrm{p}<0.01)$. These two consultation factors were included in the regression model as confounding effects without interaction with the two intervention arms.

\section{Impact of the two interventions on question asking}

The effect of the hierarchical structure of our sample (patients nested within doctor and doctor nested within centres) was measured by intra-class correlation (ICC) which measures the proportion of the total variability explained by the between-level variance. The results of this analysis revealed that ICC for centre $=0.08$ and ICC (doctor I centre) $=0.12$ respectively. (For details see figure 2). Due to these moderate ICG values ${ }^{33}$ (more than $5 \%$ ) we utilised multilevel techniques in the regression model.

The 158 patients in the QPS group selected an average of 22 questions $(\mathrm{SD}=12$, range $0-50$ ) each, for an overall total of 3392 questions. The 150 patients in the QL group self generated an average of of 2.4 questions $(\mathrm{SD}=2.3$, range 0-16) for an overall total of 293 questions.

Patients in the QPS group asked their physicians a total of 259 of the their 3392 questions $(8 \%)$. Patients in the QL group asked a total of $133(45 \%)$ of their questions

Five patients in the QPS group selected no questions and 43 patients in the QL group did generate any of their own questions.
Patients in the QPS group asked an average of 13 questions $(\mathrm{SD}=9.0)$ compared with an average of 16 questions in the $\mathrm{QL}$ group $(\mathrm{SD}=12.4)$. The multilevel regression estimated this non-significant difference at 1.7 (CI -0.3 to $3.6, \mathrm{p}=0.10)$, holding the confounding variables constant: the consultation length $(\mathrm{b}=0.3$, CI 0.2 to $0.3, \mathrm{p}<0.01)$ and the presence of companions $(\mathrm{b}=$

-4.9 , CI -7.2 to $-2.7, \mathrm{p}<0.01$ ). Thus, (1) a patient enrolled in the QL group asked, on average, 1.7 more questions than a patient enrolled in the QPS group, (2) the presence of a companion reduced the number of patient's questions asked (about 5) and (3) that despite their assigned group, on average, patient asked more questions during longer consultations (a new question every $3 \mathrm{~min}$ ).

Regarding question topics, as shown in table 2, patients most commonly asked about illness management $(41.2 \%)$ and administrative issues $(32.5 \%)$. There were no significant differences between the two groups.

\section{Satisfaction with information}

We observed significant differences between groups in satisfaction with the information. Patients with the QPS asked less of their selected questions $(t=3.60 ; p<0.01)$ compared with the QL group and were significantly less satisfied with information they received $(\mathrm{t}=2.38$; $\mathrm{p}<0.05)$. Compared with the QL group, patients in the 
Table 2 Patients questions by topic

\begin{tabular}{|c|c|c|c|c|c|c|}
\hline & \multicolumn{2}{|l|}{ QPS } & \multicolumn{2}{|l|}{ QL } & \multicolumn{2}{|c|}{ Wilcoxon test } \\
\hline & M & (SD) & $\mathbf{M}$ & (SD) & z-test & p-Value \\
\hline Symptoms & 1.74 & $(1.86)$ & 2.32 & (3.07) & -0.46 & 0.65 \\
\hline Aetiology & 0.18 & $(0.58)$ & 0.31 & $(0.78)$ & -1.79 & 0.07 \\
\hline Prognosis & 0.45 & $(0.80)$ & 0.67 & $(1.27)$ & -1.14 & 0.25 \\
\hline Prevention & 0.94 & (1.49) & 1.09 & $(1.60)$ & -0.60 & 0.55 \\
\hline IIIness Management & 5.75 & $(4.88)$ & 6.29 & (5.48) & -0.79 & 0.43 \\
\hline Administrative & 4.34 & (3.44) & 5.18 & $(4.37)$ & -1.25 & 0.21 \\
\hline
\end{tabular}

M, mean; QL, question list; QPS, question prompt sheet.

QPL group reported less need for information $(t=2.20$; $\mathrm{p}<0.05)$.

\section{Satisfaction with the decision and anxiety}

Patient levels of satisfaction with the decision were equally high in both experimental groups. Pre-consultation anxiety levels were also equal between the two groups. After the consultation, anxiety levels decreased but were not significantly different between groups (table 3 ).

\section{DISCUSSION}

Patient question asking is now considered an essential component of patient centred communication during physician - patient consultations. Our knowledge of optimal methods to promote question asking is evolving. It is important to compare the efficacy of methods of promoting question asking and the utility of different methods to improve patient outcomes.

In this RCT we hypothesised that patients who had selected their question from an evidence based set of questions, available on the QPS, would ask a greater number of questions than patients who self-generated their own list of questions on the QL. After taking into account consultation length and the presence of companion as confounders, we observed no statistical difference in question asking between the two groups. Also regarding the question topics, there were no statistical differences. However patients who utilised the QPS stated that they had been less able to ask questions they had previously selected, and were less satisfied with the information they received, even though they had no more additional questions they want to ask when compared with the QL group. No differences between groups were found regarding the level of satisfaction with decision and the level of anxiety.

Previous studies in the field ${ }^{10-13}$ 15-19 $^{19}$ showed that, compared with a standard care control group, the use of either a Question Prompt Sheet or Question Listing improved question asking.

Counter to our hypothesis it is noteworthy that the use of the QPS and the use of the QL resulted an equal number of overall questions asked. This suggests that both our interventions, administered without a coaching session and not brought into the consultation, are equally important to prepare patients to ask questions during their consultation. Providing space before the consultation can

Table 3 Questionnaires

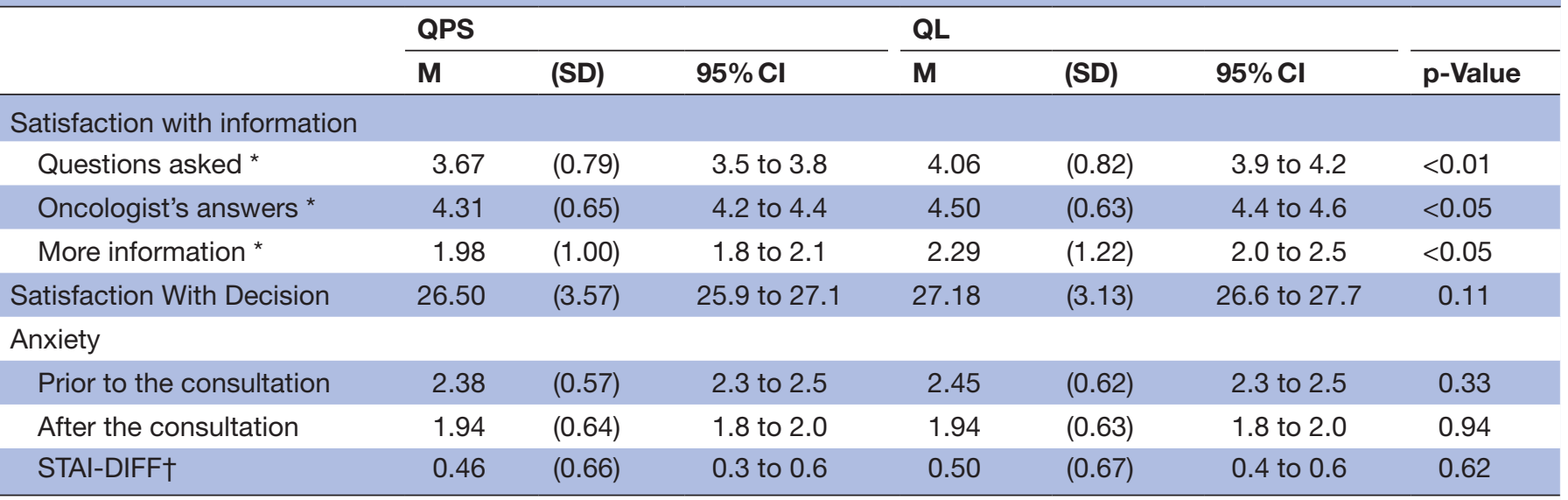

"Mean score of 'How much do you feel the need for further information?'

"Mean score of 'How much have you been able to ask those questions you had previously selected on the QPS?/ written in the QL?'

*Mean score of 'How much did the oncologist answer in a clear and comprehensive way?'

†The STAI-DIFF was usually calculated on total score, here we report the mean score

M, mean; QL, question list; QPS, question prompt sheet; STAI-DIFF: State-Trait Anxiety Inventory-Difference. 
help patients to focus on their own information needs and think about what they want to ask their doctor.

In our sample, the QPS might have reduced the number of questions compared with QL by encouraging patients to ask more focused and precise questions. This assumption can be supported by the results from a previous observational study ${ }^{34}$ conducted by our group in the same context (ie, with patients recruited with the same procedure and with the same clinical and socio-demographic characteristics), showing that patients without question asking interventions prior to the consultation ask a mean of 18 questions (slightly more than 13 questions in this sample). This observation highlights the importance of question phrasing as important to promote salient question asking.

Our finding although not significant, that patients in the QL group asked more question than those in the QPS group was counter-intuitive. It may be that the number of questions asked could be mediated by the patient's recall ability. Thus, patients who self-generate question using the QL may produce fewer questions and the generated questions may be phrased in salient terms that are easier for patients to recall. In the absence of the QPS/QL to take into the consultation, it may be that QPS patients had more difficulty remembering their desired questions than patients in the QL group. This could be supported by the fact that patients in the QL group asked more of the questions that they had previously written (133 out of 293) compared with patients in the QPS group, who asked fewer questions (259 out of 3392). It is possible that patients in the QPS felt somewhat constrained by the questions they had to choose from and therefore asked fewer questions that reflect the priority needs. However, these results should be deeply analysed, taking into consideration the quality of the communication exchange, exploring whether the physician has provided information without the patient's request and how the physician answer the patient.

Moreover, in the absence of the QPS/QL to take into the consultation, oncologists may have taken the lead in determining the course of the medical consultation, leaving little space for patients to take sufficiently active question asking role in the consultation.

Our finding that consultation length was a confounder suggests that Italian oncologists utilise the time spent in consultation somewhat differently than oncologists from other Western medical cultures. The average length of Italian consultation in oncology seems to be slightly longer, 49 compared with $30-39$ min reported by other. ${ }^{15} 1726$ Since the number of questions noted, regardless of intervention groups is quite similar to other studies ${ }^{15-19}$ it may be that Italian oncologists spend more time giving information when answering patient questions. A QPS might be more effective in enhancing recall of information when the provider receives training in how to endorse it and how to encourage patients to ask questions from it. ${ }^{35} 36$

It has been demonstrated in English speaking countries that a QPS is a useful tool to improve patient's participation during the consultation. However, we contend that consultation communication may vary across cultures and thus there is the need to explore the efficacy of a QPS intervention in Non-English speaking countries to better explore cross-cultural differences. ${ }^{36}$

We feel this study contributes to move our scientific knowledge forward by increasing our knowledge of cultural influences on question asking and on doctor-patient communication. More research is needed to improve a mutual understanding between countries leading to a more fruitful doctor-patient interaction and implementing a more effective co-operation and integration of healthcare policies.

Due to the complexity of the interaction between the patient and the oncologist, focusing on the patient alone may not produce short and long-term benefits for patients. It is plausible that oncologists, trained specifically to respond to more active patients, can further enhance patient involvement and lead to a more successful consultation. Recent studies have observed limited skills of the oncologists in shared decision-making claiming for the need of specific training to implement patient involvement in clinical practice. ${ }^{3738}$ There is the need to improve such skills but we need to know the differences between countries to generate a powerful and focused training programme. For this reason future research is warranted to explore cross cultural differences and the role of the oncologists to improve patient involvement.

\section{Limitation, strengths and future direction}

This study has some limitations to be considered. First of all, in order to ensure that the oncologists were blind to their patient experimental group assignment, we deviated from the accepted intervention process and did not provide copies of the completed QPS or QL for patients to use during their consultation to serve as a reminder to ask questions.

Furthermore, even if this study compared two active interventions (QPS vs. QL) and ensured participants blinding, the administration of the QL has been done without any previous coaching session as it was in some previous studies. In order to make the two intervention more similar and comparable the QL was manage independently by the patients.

Of note, our sample was larger than most previous studies in the field ${ }^{13161819}$ and large enough to generalise our results to early stage female breast cancer patients with access to similar health services in Northern Italy. As far as we know, this is also the first study in Italy analysing audiotapes of routine oncological consultations, an approach which has inherent ecological validity and that is a recommended research tool ${ }^{339}$ which allow future analysis on information exchange.

\section{Conclusions}

Findings from the present study suggested that QPS have a similar impact on question asking compared with QL, if completed independently by the patients. Both could be 
useful tools for stimulating patient's participation during the consultation. Maybe the opportunity to rehearse their information needs before the consultation can help patients to better focus on them and to use the time spent during the consultation more efficiently, asking questions to their doctor that are more precise. This should be explored deeply in future studies along with the exploration of which components of the interventions, focused on increasing patient participation, are really useful and efficacious. These interventions are relatively simple and inexpensive to disseminate in routine care with limited disruption to clinic flow.

Even if we did not found variables associated to question asking, some patients might find the interventions more helpful than others. Further research is also needed to identify those patients to whom intervention are likely to be most beneficial and the role of the oncologist in leading the consultations.

\section{Author affiliations}

${ }^{1}$ Department of Neurological, Biomedical and Movement Sciences, Section of Clinical Psychology, University of Verona, Verona, Italy

${ }^{2}$ Department of Clinical and Experimental Sciences, Section of Clinical Psychology, University of Brescia, Brescia, Italy

${ }^{3}$ Operative Unit of Medical Oncology, Ospedale Civile Maggiore, Hospital Trust of Verona, Verona, Italy

${ }^{4}$ Operative Unit of Medical Oncology, Policlinico G. Rossi, Hospital Trust of Verona, Verona, Italy

${ }^{5}$ Operative Unit of Medical Oncology, Spedali Civili, Brescia, Italy

${ }^{6}$ Department of Health Behavior and Policy, School of Medicine, Virginia

Commonwealth University, Richmond, USA

Acknowledgements Wegratefullyacknowledge the participation of allpatients and the contribution of the medicaloncologistsat the Operative Units of the three centres involved, including Annamaria Molino, Elena Fiorio, Veronica Parolin, Michele Pavarana, and Francesca La Russa (Ospedale Civile Maggiore, Verona); Rolando Nortilli, Federica Maspero, Chiara Caliolo, Alessandra Auriemma, Serena Zuliani, Jenni Furlanetto and Francesca Maines (Policlinico G. Rossi, Verona); Vito Amoroso, Lucia Vassalli, Francesca Valcamonico, Salvatore Grisanti, Francesca Consoli, Rebecca Pedersini, and Laura Deiana (Spedali Civili, Brescia). We also thank Christa Zimmermann for reviewing the manuscript, and Mario Ballarin for his contribution in developing the database for data storage and retention. Finally we want to thanks Lara Ferretto, Silvia Tregnago, MicolMeneghini, Veronica Fremondi, DomenicoBarbera, and Paola Mazzardi who assisted with patient recruitment, coding and data collection.

Contributors $A B$ involved in the data acquisition, data analysis, interpretation, drafting/critical revision of the work and approved the final manuscript. CG involved in the conception, design, interpretation, drafting/critical revision of the work and approved the final manuscript. MAM involved in data analysis, critical revision, and approved the final manuscript. AG involved in the conception, critical revision, and approved the final manuscript. CB involved in the data acquisition and critical revision, and approved the final manuscript. AM involved in the data acquisition and approved the final manuscript. EF involved in the data acquisition and approved the final manuscript. RN involved in the data acquisition and approved the final manuscript. VA involved in the data acquisition and approved the final manuscript. LV involved in the data acquisition and approved the final manuscript. RFB involved in the interpretation, drafting/critical revision of the work and approved the final manuscript.

Funding This research received no specific grant from any funding agency in the public, commercial or not-for-profit sectors.

Competing interests None declared.

Ethics approval Ethics Committee of the Hospital Trust of Verona and the Provincial Ethics Committee of Brescia.

Provenance and peer review Not commissioned; externally peer reviewed.
Data sharing statement $A$ dataset (Excel format) is available as supplementary material.

Open Access This is an Open Access article distributed in accordance with the Creative Commons Attribution Non Commercial (CC BY-NC 4.0) license, which permits others to distribute, remix, adapt, build upon this work non-commercially, and license their derivative works on different terms, provided the original work is properly cited and the use is non-commercial. See: http://creativecommons.org/ licenses/by-nc/4.0/

C) Article author(s) (or their employer(s) unless otherwise stated in the text of the article) 2017. All rights reserved. No commercial use is permitted unless otherwise expressly granted.

\section{REFERENCES}

1. Jenkins V, Fallowfield L, Saul J. Information needs of patients with Cancer: results from a large study in UK Cancer centres. $\mathrm{Br} J$ Cancer 2001;84:48-51.

2. Del Piccolo L, Goss C. People-centred care: new research needs and methods in doctor-patient communication. challenges in mental health. Epidemiol Psychiatr Sci 2012;21:145-9.

3. Butow PN, Dowsett S, Hagerty R, et al. Communicating prognosis to patients with metastatic disease: what do they really want to know? Support Care Cancer 2002;10:161-8.

4. Eggly S, Penner LA, Greene M, et al. Information seeking during "bad news" oncology interactions: Question asking by patients and their companions. Soc Sci Med 2006;63:2974-85.

5. Ellis PM, Tattersall MH. How should doctors communicate the diagnosis of Cancer to patients? Ann Med 1999;31:336-41.

6. Lenzi R, Baile WF, Berek J, et al. Design, conduct and evaluation of a communication course for oncology fellows. J Cancer Educ 2005;20:143-9.

7. Siminoff LA, Ravdin P, Colabianchi N, et al. Doctor-patient communication patterns in breast Cancer adjuvant therapy discussions. Health Expect 2000;3:26-36.

8. Street RL. Active patients as powerful communicators. In: Robinson WP, Giles H, eds. The new handbook of language and social psychology.. New York: John Wiley \& Sons, 2001:541-60.

9. Bensing J, Verhaak P. Communication in medical encounters. Malden, MA, US: Blackwell Publishing, 2004.

10. Jones R, Pearson J, McGregor S, et al. Does writing a list help Cancer patients ask relevant questions? Patient Educ Couns 2002;47:369-71.

11. Belkora JK, Loth MK, Chen DF, et al. Monitoring the implementation of Consultation Planning, Recording, and summarizing in a breast care center. Patient Educ Couns 2008;73:536-43.

12. Kidd J, Marteau TM, Robinson S, et al. Promoting patient participation in consultations: a randomised controlled trial to evaluate the effectiveness of three patient-focused interventions. Patient Educ Couns 2004;52:107-12.

13. Sepucha KR, Belkora JK, Tripathy D, et al. Building bridges between physicians and patients: results of a pilot study examining new tools for collaborative decision making in breast Cancer. J Clin Oncol 2000;18:1230-8.

14. Belkora J, Katapodi M, Moore D, et al. Evaluation of a visit preparation intervention implemented in two rural, underserved counties of Northern California. Patient Educ Couns 2006;64:350-9.

15. Brown RF, Butow PN, Dunn SM, et al. Promoting patient participation and shortening Cancer consultations: a randomised trial. $\mathrm{Br} \mathrm{J}$ Cancer 2001;85:1273-9.

16. Butow PN, Dunn SM, Tattersall MH, et al. Patient participation in the Cancer consultation: evaluation of a question prompt sheet. Ann Oncol 1994;5:199-204.

17. Clayton JM, Butow PN, Tattersall MH, et al. Randomized controlled trial of a prompt list to help advanced Cancer patients and their caregivers to ask questions about prognosis and end-of-life care. $J$ Clin Oncol 2007;25:715-23.

18. Brown R, Butow PN, Boyer MJ, et al. Promoting patient participation in the Cancer consultation: evaluation of a prompt sheet and coaching in question-asking. Br J Cancer 1999;80:242-8.

19. Bruera E, Sweeney C, Willey J, et al. Breast Cancer patient perception of the helpfulness of a prompt sheet versus a general information sheet during outpatient consultation: a randomized, controlled trial. J Pain Symptom Manage 2003;25:412-9.

20. Roter DL. Patient participation in the patient-provider interaction: the effects of patient question asking on the quality of interaction, satisfaction and compliance. Health Educ Monogr 1977;5:281-315. 
21. Kinnersley P, Edwards A, Hood K, et al. Interventions before consultations to help patients address their information needs by encouraging question asking: systematic review. BMJ 2008;337:a485.

22. Leurent B, Reyburn H, Muro F, et al. Monitoring patient care through health facility exit interviews: an assessment of the Hawthorne effect in a trial of adherence to malaria treatment guidelines in Tanzania. BMC Infect Dis 2016;16:59.

23. Moher D, Hopewell S, Schulz KF, et al. CONSORT 2010 explanation and elaboration: updated guidelines for reporting parallel group randomised trials. BMJ 2010;340:c869.

24. Goss C, Ghilardi A, Deledda G, et al. INvolvement of breast CAncer patients during oncological consultations: a multicentre randomised controlled trial--the INCA study protocol. BMJ Open 2013;3:e002266.

25. Street RL, Gordon H, Haidet P. Physicians' communication and perceptions of patients: is it how they look, how they talk, or is it just the doctor? Soc Sci Med 2007;65:586-98.

26. Butow P, Devine R, Boyer M, et al. Cancer consultation preparation package: changing patients but not physicians is not enough. $J$ Clin Oncol 2004;22:4401-9.

27. Cancer Institute NSWSidney AUS. Medical/Radiating Oncologist Question list. https://www.cancerinstitute.org.au/getattachment/ 27b46c49-b480-4517-90bc-cc6c1317b2af/2009-06-15_question list_oncologist.pdf (accessed 2015 Nov 12).

28. Holmes-Rovner M, Kroll J, Schmitt N, et al. Patient satisfaction with health care decisions: the satisfaction with decision scale. Med Decis Making 1996;16:58-64.

29. Speilberger CD. Manual for the State trait anxiety inventory for adults (Form Y. Palo Alto, CA: Mind Garden, 1983.
30. Sanavio E. Le Scale CBA. Milano: Raffaello Cortina Editore, 2002.

31. Sanavio E, Bertolotti G, Bettinardi O, et al. The Cognitive Behavioral Assessment (CBA) Project: presentation and proposal for International Collaboration. Psychology, Community \& Health 2013;2:362-80.

32. Skrondal A, Rabe-Hesketh S. Generalized latent variable modeling: multilevel, longitudinal, and structural equation models. New York: Chapman \& Hall/CRC, 2004

33. Hox JJ. Applied multilevel analysis: techniques and applications. Mahwah, NJ: Lawrence Erlbaum Associates Inc, 2002.

34. Goss C, Deledda G, Bottacini A, et al. Information needs of female italian breast Cancer patients during their first oncological consultation. Eur J Oncol Nurs 2015;19:451-7.

35. Brandes K, Linn AJ, Butow PN, et al. The characteristics and effectiveness of Question Prompt list interventions in oncology: a systematic review of the literature. Psychooncology 2015;24:245-52.

36. Sansoni JE, Grootemaat P, Duncan C. Question prompt lists in health consultations: a review. Patient Educ Couns 2015;98:1454-64.

37. Kunneman M, Engelhardt EG, Ten Hove FL, et al. Deciding about (neo-)adjuvant rectal and breast Cancer treatment: missed opportunities for shared decision making. Acta Oncol 2016;55:134-9.

38. Müller E, Hahlweg P, Scholl I. What do stakeholders need to implement shared decision making in routine Cancer care? A qualitative needs assessment. Acta Oncol 2016;55:1484-91.

39. Tattersall MH, Butow PN. Consultation audio Tapes: an underused Cancer patient information aid and clinical research tool. Lancet Oncol 2002;3:431-7. 